\title{
ANÁLISE DE MODO E EFEITOS DE FALHA: UM ESTUDO BIBLIOMÉTRICO NA BASE WEB OF SCIENCE
}

\section{REVISÃO BIBLIOMÉTRICA}

LOYOLA, Vagner Viali ${ }^{1}$

XAVIER, Thiago Padovani ${ }^{2}$

LOYOLA, Vagner Viali. XAVIER, Thiago Padovani. Análise de Modo e Efeitos de Falha: Um estudo bibliométrico na base Web of Science. Revista Científica Multidisciplinar Núcleo do Conhecimento. Ano 05, Ed. 10, Vol. 09, pp. 193-208. Outubro de 2020. ISSN: 2448-0959, Link de acesso: https://www.nucleodoconhecimento.com.br/engenharia-de-producao/efeitosde-falha

\section{RESUMO}

O presente artigo analisa as características das publicações relacionadas ao tema FMEA (Failure Mode and Effects Analysis - Análise de Modo e Efeitos de Falha). A pesquisa foi realizada na base de dados Web of Science, no período compreendido entre 2009 e 2019, procurando identificar: número e ano das publicações, idiomas, autores, organizações, áreas de pesquisa, países, agências financiadoras, títulos da fonte e o número de citações dos artigos encontrados. De acordo com os resultados encontrados, há poucos estudos publicados no período analisado, 290 publicações, sendo China, Estados Unidos e Brasil os países com maior número de registros. Ademais, cerca de $93 \%$ encontram-se no idioma inglês.

Palavras-chave: FMEA, Pesquisa Bibliométrica.

${ }^{1}$ Graduando em Engenharia de Produção.

2 Orientador. Doutor em Engenharia Química. 


\section{INTRODUÇÃO}

Com o aumento da globalização e da competição entre empresas, o sucesso dos projetos de uma organização se tornou ainda mais decisivo para o desenvolvimento do negócio da mesma. Desse modo, é necessário que haja uma gestão eficaz dos riscos nesses projetos, para que os mesmos não gerem atrasos ou sejam cancelados por não serem viáveis. Entretanto, ferramentas de gerenciamento desses riscos são pouco empregadas em organizações de diversos setores empresariais.

Algumas aplicações práticas sobre gerenciamento de riscos de projetos podem ser encontradas em: Júnior e Carvalho (2013), Ferenhof, Forcellini, Varvakis (2013), Piurcosky et al. (2014), Sena et al. (2014) e Espósito (2015).

De modo a diminuir os impactos dos riscos negativos, metodologias, ferramentas e técnicas devem ser utilizadas, para uma maior identificação e eliminação dos mesmos, sendo que uma das ferramentas utilizadas no meio empresarial para a análise e gerenciamento de riscos é a FMEA (Failure Mode and Effects Analysis Análise de Modo e Efeitos de Falha), pois, permite a detecção de possíveis falhas em um processo ou produto, averiguando todos os seus componentes, além de analisar todos os efeitos das falhas na confiabilidade, traçando planos de ação que determinem as providências a serem tomadas.

O FMEA surgiu por volta de 1949 e destinava-se às análises de falhas em sistemas e equipamentos do exército americano, onde era avaliada a sua eficiência baseando-se no impacto sobre uma missão ou no sucesso de defesa pessoal de cada soldado. Desde 1976, esse método vem sendo usado no ramo automobilístico e atualmente constitui-se numa ferramenta imprescindível para as empresas fornecedoras deste segmento (DAILEY, 2004).

A partir dessa breve contextualização, o presente estudo teve como objetivo analisar as características das publicações referentes à essa ferramenta junto à base de dados Web of Science, no período compreendido entre 2009 e 2019. 


\section{METODOLOGIA}

O presente estudo desenvolveu-se a partir de uma pesquisa bibliométrica, com o objetivo de aumentar o conhecimento de publicações relacionadas ao tema FMEA.

A bibliometria, segundo Silva (2004), analisa a atividade científica ou técnica por meio do estudo quantitativo das publicações, desse modo, o estudo possui uma abordagem quantitativa, pois procurou quantificar variáveis referentes à produção científica sobre a ferramenta FMEA.

Para a realização desta pesquisa, os dados foram coletados na base Web of Science do Institute for Scientific Information (ISI), que consiste em uma base multidisciplinar que disponibiliza os periódicos mais citados em suas respectivas áreas, permitindo identificar as citações recebidas, as referências utilizadas e os registros relacionados, assim como a criação de rankings por inúmeros parâmetros, possuindo mais de 12.000 periódicos indexados (CAPES, 2012).

Com isso, a partir do mecanismo de busca da Web of Science, utilizando como palavra-chave FMEA e delimitando a busca para o período de 2009 a 2019, as publicações foram pesquisadas para sua posterior análise.

Inicialmente, foi inserido a palavra FMEA como tópico no campo de pesquisa da base Web of Science, utilizando como parâmetros o período de 2009 a 2019 e artigos de acesso aberto. A partir dos resultados encontrados, o desenvolvimento da pesquisa baseou-se em análise gráfica e tabelas feitas com o auxílio do Microsoft Excel e o software VOSviewer. Desse modo, foram verificadas informações relacionadas ao número total de publicações, idiomas, autores, países, organizações, ano das publicações, áreas de pesquisa, agências financiadoras, títulos da fonte, o número de citações anual dos artigos e a co-ocorrência de palavras-chave relacionadas ao tema. Com isso, a análise bibliométrica do estudo foi realizada, sendo apresentada na seção seguinte. 


\section{ANÁLISE E DISCUSSÃO DOS RESULTADOS}

Foram encontrados 290 artigos relacionados ao tema FMEA, no período especificado, sendo que $93 \%$, aproximadamente, se encontram no idioma inglês. A seguir, serão apresentadas as informações relativas à essas publicações, sendo que as mesmas serão apresentadas de acordo com as seguintes categorias: áreas de pesquisa, ano das publicações, organizações, países, agências financiadoras, autores, títulos da fonte, número de citações e co-ocorrência de palavras-chave.

\section{1 ÁREAS DE PESQUISA}

A Tabela 1 mostra as principais áreas de pesquisa relacionadas ao tema pesquisado. Por meio dela, é possível perceber que a Engenharia possui um maior número de publicações sobre a ferramenta FMEA, com 105 artigos, aproximadamente 36\%, seguida das outras áreas que apresentam um número abaixo considerável. Isso mostra que apesar de ser uma ferramenta que pode ser adotada em diversas áreas, a Engenharia apresenta um maior estudo e aplicação da mesma.

Tabela 1. Áreas de pesquisa.

\begin{tabular}{|l|l|}
\hline Áreas & Quantidade de artigos \\
\hline Engenharia & 105 \\
\hline Ciência da computação & 36 \\
\hline Radiologia nuclear e medicina com imagens médicas & 22 \\
\hline Economia de negócios & 17 \\
\hline Ciência de materiais & 16 \\
\hline Ecologia das ciências ambientais & 15 \\
\hline Ciência, tecnologia, outros tópicos & 14 \\
\hline Combustíveis de energia & 13 \\
\hline Matemática & 12 \\
\hline Saúde ocupacional ambiental pública & 12 \\
\hline Física & 11 \\
\hline
\end{tabular}

RC: 63222

Disponível em: https://www.nucleodoconhecimento.com.br/engenharia-de-producao/efeitos-de$\underline{\text { falha }}$ 


\begin{tabular}{|l|l|}
\hline Telecomunicações & 11 \\
\hline Transporte & 11 \\
\hline Serviços de ciências da saúde & 10 \\
\hline Outras áreas & 125 \\
\hline
\end{tabular}

Fonte: Elaborado pelo autor (2020).

\subsection{PUBLICAÇÕES POR ANO}

No período analisado, observou-se que entre os anos de 2009 e 2014 poucos artigos foram publicados, tendo um crescimento a partir de 2015 e chegando à 68 publicações no ano de 2019. A Figura 1 mostra esse fato.

Figura 1. Publicações por ano.

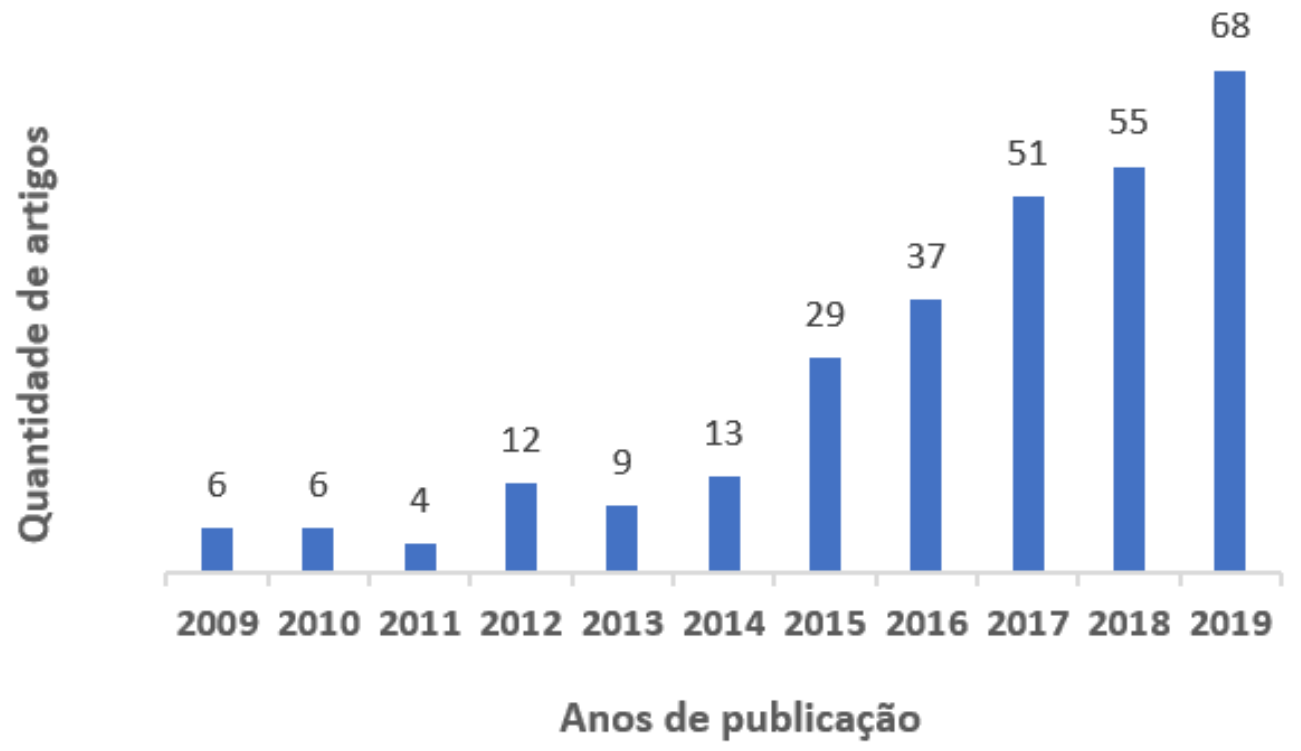

Fonte: Elaborado pelo autor (2020).

Por meio dela, fica evidente como esse tema foi pouco estudado por autores ao longo desses anos, sendo que o ano de 2019 registrou o maior número de publicações, 68 artigos. Isso mostra que essa ferramenta da qualidade é relativamente nova para a análise de falha de diversos processos em diferentes áreas, sendo necessário uma

Disponível em: https://www.nucleodoconhecimento.com.br/engenharia-de-producao/efeitos-de$\underline{\text { falha }}$ 
maior pesquisa e conhecimento sobre o tema para que, cada vez mais, novos trabalhos sejam produzidos.

\subsection{ORGANIZAÇÕES}

As instituições que mais publicaram artigos relacionados ao tema FMEA estão apresentadas na Tabela 2.

Por meio dela, é possível perceber que a instituição que mais se destaca no que se refere às publicações referentes à pesquisa realizada é a Universidade de Tecnologia da Silésia, localizada na Polônia. Apesar disso, outras organizações possuem números próximos à essa Universidade, demonstrando que várias instituições estão pesquisando sobre essa ferramenta que é muito importante para a análise de risco de um produto ou processo de uma empresa.

Tabela 2. Principais organizações.

\begin{tabular}{|l|l|}
\hline Organizações & $\begin{array}{l}\text { Quantidade de } \\
\text { artigos }\end{array}$ \\
\hline Universidade de Tecnologia da Silésia & 9 \\
\hline Universidade de Malásia Sarawak & 8 \\
\hline Universidade Deakin & 7 \\
\hline Universidade Politécnica do Noroeste & 7 \\
\hline Universidade de Tecnologia de Ciência Eletrônica da & 6 \\
\hline China & \\
\hline Universidade de Chongqing & 5 \\
\hline Universidade de Tecnologia Amirkabir & 4 \\
\hline Sistema de Educação Superior da Comunidade da & 4 \\
\hline Pensilvânia & \\
\hline Universidade de Tecnologia Tallinn & 4 \\
\hline Universidade Técnica Kosice & 4 \\
\hline Universidade Tianjin & 4 \\
\hline
\end{tabular}

Disponível em: https://www.nucleodoconhecimento.com.br/engenharia-de-producao/efeitos-defalha 


\begin{tabular}{|l|l|}
\hline Universidade de Milão & 4 \\
\hline Universidade de Pittsburgh & 4 \\
\hline Universidade de Ciências das Artes de Chongqing & 3 \\
\hline Universidade Cranfield & 3 \\
\hline CTR Geral Reprod Med & 3 \\
\hline Universidade Johns Hopkins & 3 \\
\hline Universidade de Liverpool John Moores & 3 \\
\hline Universidade de Stanford & 3 \\
\hline Universidade Técnica de Berlim & 3 \\
\hline Universidade de Ciências Médicas de Teerã & 3 \\
\hline Universidade Tongji & 3 \\
\hline Universidade de São Paulo & 3 \\
\hline Universidade de Teerã & 3 \\
\hline
\end{tabular}

Fonte. Elaborado pelo autor (2020).

\subsection{PAÍSES}

Em relação aos principais países que possuem publicações relacionadas à pesquisa, a Tabela 3 apresenta os resultados encontrados.

É possível perceber que a China é o país que mais se destaca com 48 registros, seguida dos Estados Unidos e Brasil, com 31 e 22 registros, respectivamente. É importante salientar que, apesar de o Brasil estar em terceiro no ranking dos principais países, não há organizações entre as primeiras que mais realizaram estudos, como mostrado na Tabela 2, sendo a Universidade de São Paulo a primeira a aparecer no ranking com 3 artigos.

Por outro lado, a Polônia que apresenta a Universidade de Tecnologia da Silésia como a organização que mais publica artigos sobre a ferramenta estudada, é apenas o sexto país com 19 registros. 
Tabela 3. Países que mais publicam.

\begin{tabular}{|l|l|}
\hline Países & Quantidade de artigos \\
\hline China & 48 \\
\hline Estados Unidos & 31 \\
\hline Brasil & 22 \\
\hline Irã & 21 \\
\hline Inglaterra & 20 \\
\hline Polônia & 19 \\
\hline Itália & 15 \\
\hline Malásia & 14 \\
\hline Turquia & 12 \\
\hline Espanha & 11 \\
\hline Taiwan & 10 \\
\hline Austrália & 9 \\
\hline Alemanha & 8 \\
\hline
\end{tabular}

Fonte: Elaborado pelo autor (2020).

\subsection{AGÊNCIAS FINANCIADORAS}

$\mathrm{Na}$ Tabela 4 estão apresentadas as agências financiadoras de pesquisas relacionadas ao tema pesquisado. A partir dela, é possível perceber que a Fundação Nacional de Ciências Naturais da China é a que mais se destaca com 29 estudos financiados. Fundada em 14 de fevereiro de 1986, é uma organização diretamente afiliada ao conselho de Estado da China, atuando na administração do Fundo Nacional de Ciências Naturais.

Ademais, o Departamento de Serviços Humanos e Saúde dos Estados Unidos é o segundo em relação à quantidade de artigos, com 9 registros, o que justifica o fato de China e Estados Unidos serem os países que mais realizam estudos sobre o tema, conforme a Tabela 3. 
Tabela 4. Agências financiadoras.

\begin{tabular}{|c|c|}
\hline Agências Financiadoras & $\begin{array}{l}\text { Quantidade de } \\
\text { artigos }\end{array}$ \\
\hline Fundação Nacional de Ciências Naturais da China & 29 \\
\hline $\begin{array}{l}\text { Departamento de Serviços Humanos e Saúde dos Estados } \\
\text { Unidos }\end{array}$ & 9 \\
\hline Instituto Nacional de Saúde Nih dos Estados Unidos & 7 \\
\hline $\begin{array}{l}\text { Fundos de Pesquisa Fundamental para as Universidades } \\
\text { Centrais }\end{array}$ & 5 \\
\hline FRGS Grant & 5 \\
\hline Ministério da Educação e Pesquisa da Estônia & 4 \\
\hline $\begin{array}{l}\text { Plano de Pesquisa Básica de Ciências Naturais da Província } \\
\text { de Shaanxi da China }\end{array}$ & 4 \\
\hline $\begin{array}{l}\text { Conselho de Pesquisa de Ciências Físicas de Engenharia } \\
\text { EPSRC }\end{array}$ & 3 \\
\hline União Europeia UE & 3 \\
\hline Race Grant & 3 \\
\hline Fundação de Ciência da Aviação & 2 \\
\hline $\begin{array}{l}\text { Programa de Fundo de Doutorado para Inovação da } \\
\text { Universidade do Sudoeste de Jiaotong }\end{array}$ & 2 \\
\hline Esquema de Concessão de Pesquisa Exploratória & 2 \\
\hline Governo Coreano & 2 \\
\hline $\begin{array}{l}\text { Ministério de Educação Esportes Juvenis da República } \\
\text { Tcheca }\end{array}$ & 2 \\
\hline Ministério da Ciência e Tecnologia de Taiwan & 2 \\
\hline $\begin{array}{l}\text { Principal projeto nacional científico e tecnológico especial } \\
\text { para CNC de alto grau e equipamentos básicos de } \\
\text { manufatura da China }\end{array}$ & 2 \\
\hline
\end{tabular}




\section{Fundação Nacional de Ciências Naturais da Província de 2 Guangdong}

Fundação Nacional de Pesquisa da Coreia NRF Governo 2 Coreano MSIT

Conselho Nacional de Ciências de Taiwan 2

Instituto Nacional de Câncer nih NCl 2

Projeto de inovação do $10^{\circ}$ aluno de graduação da 1

Universidade de Recursos Hídricos e Energia Elétrica do

Norte da China

Universidade Aberystwyth

Fonte: Elaborado pelo autor (2020).

\subsection{AUTORES}

A Tabela 5 apresenta os principais autores que mais publicaram sobre a ferramenta pesquisada.

Por meio dela, é possível perceber que os autores que possuem um maior número de publicações para o período analisado, são Tay KM e Lim CP, com 8 e 7 registros, respectivamente. Esses dois autores estão ligados à Universidade de Malásia Sarawak, localizada em Kota Samarahan, Malásia e Universidade Deakin, localizada em Vitória, Austrália. Isso mostra que os autores que possuem um maior número de publicações estão ligados as Universidades que possuem um dos maiores números de estudos sobre o tema.

Tabela 5. Autores que mais publicaram sobre o tema.

\begin{tabular}{|l|l|}
\hline Autores & Quantidade de artigos \\
\hline Tay KM & 8 \\
\hline Lim CP & 7 \\
\hline Jiang W & 5 \\
\hline
\end{tabular}

Disponível em: https://www.nucleodoconhecimento.com.br/engenharia-de-producao/efeitos-defalha 


\begin{tabular}{|l|l|}
\hline Ran Y & 5 \\
\hline Zhang GB & 5 \\
\hline Cantone MC & 4 \\
\hline Deng XY & 4 \\
\hline Deng Y & 4 \\
\hline Liu H & 4 \\
\hline Veronese I & 4 \\
\hline Ge SY & 3 \\
\hline Huq MS & 3 \\
\hline Jong CH & 3 \\
\hline Li YL & 3 \\
\hline Rienzi L & 3 \\
\hline Sulakatko V & 3 \\
\hline
\end{tabular}

Fonte: Elaborado pelo autor (2020).

\subsection{TÍTULOS DA FONTE}

As principais fontes de publicações relacionadas ao FMEA estão apresentadas na Tabela 6.

É possível perceber que as revistas científicas que mais publicaram foram, Energies e IEEE Access, ambas de acesso aberto e relacionadas com a área de engenharia.

Embora sejam as duas que mais publicaram, não possuem uma grande diferença em relação às outras fontes de publicação, demonstrando que essa ferramenta é divulgada por diversas fontes devido a sua aplicabilidade em diversos processos de diferentes áreas. 
Tabela 6. Títulos da fonte.

\begin{tabular}{|l|l|}
\hline Títulos Da Fonte & $\begin{array}{l}\text { Quantidade de } \\
\text { artigos }\end{array}$ \\
\hline Energies & 9 \\
\hline IEEE Access & 9 \\
\hline Medical Physics & 8 \\
\hline Tehnicki Vjesnik Technical Gazette & 7 \\
\hline Applied Sciences Basel & 6 \\
\hline Management Systems In Production Engineering & 5 \\
\hline Mathematical Problems In Engineering & 5 \\
\hline Metalurgija & 5 \\
\hline Sustainability & 5 \\
\hline International Journal Of Computers Communications & 4 \\
\hline Control & \\
\hline Journal Of Applied Clinical Medical Physics & 4 \\
\hline Advances In Mechanical Engineering & 3 \\
\hline Advances In Production Engineering Management & 3 \\
\hline
\end{tabular}

Fonte: Elaborado pelo autor (2020).

\subsection{CITAÇÕES}

A partir da pesquisa realizada na base de dados da Web of Science, a Tabela 7 mostra as doze publicações mais citadas entre as 290 encontradas. Depois de analisada, a mesma foi comparada com a Tabela 5, dos autores que mais publicaram sobre o tema, verificando se esses foram os autores que tiveram seus artigos mais citados. 
Tabela 7. Número de citações dos artigos.

Publicações

Failure Modes and Effects Analysis (FMEA) for wind turbines

Por: Arab na Hoseynabadi, H.; Oraee, H.; Tavner, P. J.

International Journal of Electrical Power \& Energy Systems

Volume: 32 Edição: 7 Páginas: 817-824 Publicado: SEP 2010

Evaluation of safety in a radiation oncology setting using failure 121 mode and effects analysis

Por: Ford, Eric C.; Gaudette, Ray; Myers, Lee; et al.

International Journal of Radiation Oncology Biology Physics

Volume: 74 Edição: 3 Páginas: 852-858 Publicado: JUL 12009

Reliability-Centered Maintenance for Wind Turbines Based on 103 Statistical Analysis and Practical Experience

Por: Fischer, Katharina; Besnard, Francois; Bertling, Lina

IEEE Transactions on energy conversion

Volume: 27 Edição: 1 Páginas: 184-195 Publicado: MAR 2012

The report of Task Group 100 of the AAPM: Application of risk 90 analysis methods to radiation therapy quality management

Por: Huq, M. Saiful; Fraass, Benedick A.; Dunscombe, Peter B.; et al.

Medical Physics

Volume: 43 Edição: 7 Páginas: 4209-4262 Publicado: JUL 2016

Fuzzy linear programming models for new product design using 78 QFD with FMEA 
Por: Chen, Liang-Hsuan; Ko, Wen-Chang

Applied Mathematical Modelling

Volume: 33 Edição: 2 Páginas: 633-647 Publicado: FEB 2009

A systematic approach for diagnosing service failure: Service- 62 specific FMEA and grey relational analysis approach

Por: Geum, Youngjung; Cho, Yangrae; Park, Yongtae

Mathematical and Computer Modelling

Volume: 54 Edição: 11-12 Páginas: 3126-3142 Publicado: DEC 2011

Dynamic safety assessment of natural gas stations using Bayesian 58 network

Por: Zarei, Esmaeil; Azadeh, Ali; Khakzad, Nima; et al.

Journal of Hazardous Materials

Volume: 321 Páginas: 830-840 Publicado: JAN 52017

Failure mode and effects analysis outputs: are they valid?

Por: Shebl, Nada Atef; Franklin, Bryony Dean; Barber, Nick

BMC Health Services Research

Volume: 12 Número do artigo: 150 Publicado: JUN 102012

Quality by Design I: Application of Failure Mode Effect Analysis 49 (FMEA) and Plackett-Burman Design of Experiments in the Identification of "Main Factors" in the Formulation and Process Design Space for Roller-Compacted Ciprofloxacin Hydrochloride Immediate-Release Tablets

Por: Fahmy, Raafat; Kona, Ravikanth; Dandu, Ramesh; et al.

AAPS Pharmscitech

Volume: 13 Edição: 4 Páginas: 1243-1254 Publicado: DEC 2012

Disponível em: https://www.nucleodoconhecimento.com.br/engenharia-de-producao/efeitos-de- 
An FMEA-Based Risk Assessment Approach for Wind Turbine 48 Systems: A Comparative Study of Onshore and Offshore

Por: Shafiee, Mahmood; Dinmohammadi, Fateme

Energies

Volume: 7 Edição: 2 Páginas: 619-642 Publicado: FEB 2014

Failure mode and effect analysis-based quality assurance for 47 dynamic MLC tracking systems

Por: Sawant, Amit; Dieterich, Sonja; Svatos, Michelle; et al. Medical Physics

Volume: 37 Edição: 12 Páginas: 6466-6479 Publicado: DEC 2010

Application of failure mode and effects analysis to treatment 46 planning in scanned proton beam radiotherapy

Por: Cantone, Marie Claire; Ciocca, Mario; Dionisi, Francesco; et al.

\section{Radiation Oncology}

Volume: 8 Número do artigo: UNSP 127 Publicado: MAY 242013

onte: Elaborado pelo autor (2020).

Analisando as duas tabelas, foi possível perceber que apenas dois dos autores que mais publicaram no período analisado, estão entre os doze artigos mais citados, sendo eles Cantone MC e Huq MS. Isso evidencia que, as publicações com maior número de citações, que provavelmente são as de maior referência no tema pesquisado, não possuem como autores aqueles que mais publicam sobre essa ferramenta.

\subsection{PALAVRAS-CHAVE RELACIONADAS COM O TEMA}

A Figura 2 apresenta as principais palavras-chave relacionadas ao tema pesquisado. Para a realização da mesma, foi utilizado como parâmetro, palavras-chave que

Disponível em: https://www.nucleodoconhecimento.com.br/engenharia-de-producao/efeitos-defalha 
possuíam número mínimo de ocorrência igual a três, ou seja, palavras que estiveram presentes em pelo menos três artigos.

Figura 2. Rede de co-ocorrência de palavras-chave relacionadas ao FMEA.

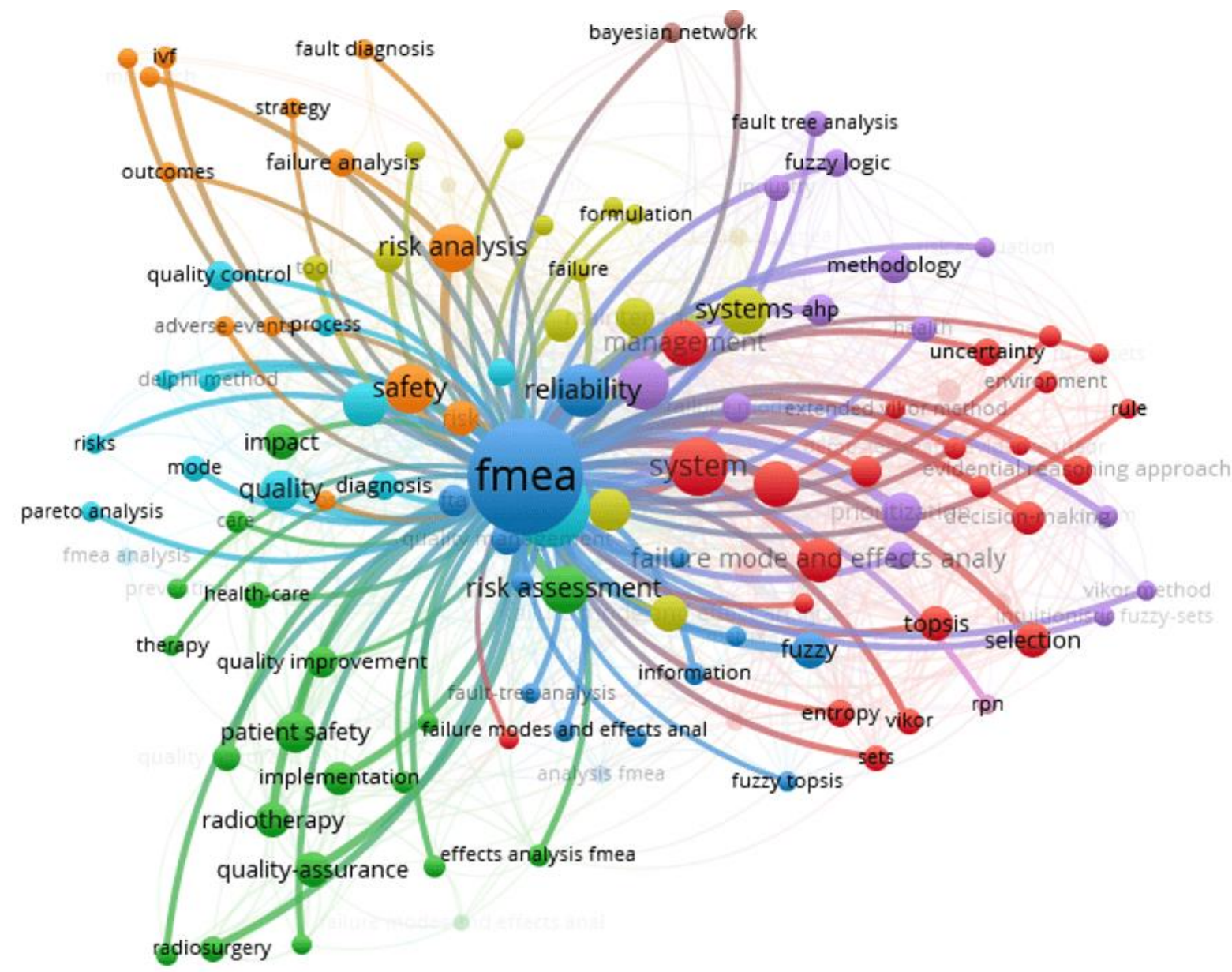

Fonte: Software VOSviewer (2020).[/caption]

De acordo com a Figura 2, as palavras-chave que mais se relacionaram ao FMEA, foram: failure mode (modo de falha), system (Sistema), reliability (Confiabilidade) e risk-evaluation (Avaliação de risco). Todas essas palavras estão relacionadas com a área de Engenharia, justificando o fato dessa área ser a principal em relação ao número de artigos publicados, além das revistas que mais possuem divulgação sobre o assunto, conforme os subtópicos 3.1 e 3.7 . 


\section{CONSIDERAÇÕES FINAIS}

A análise das publicações sobre a ferramenta da qualidade FMEA na base de dados Web of Science mostrou que 290 artigos foram encontrados no período entre 2009 e 2019, sendo que, aproximadamente, 93\% estão no idioma inglês e cerca de $36 \%$ ligados à área de Engenharia.

Observou-se que a produção científica relacionada ao tema foi relativamente baixa entre os anos analisados, tendo um crescimento a partir de 2015 e registrando, no ano de 2019, o maior número de publicações, 68 artigos. A maioria dos estudos foram publicados na China, seguida dos Estados Unidos e Brasil, sendo a Fundação Nacional de Ciências Naturais da China e Departamento de Saúde e Serviços Humanos dos Estados Unidos as agências financiadoras que mais se destacam.

Por outro lado, a Universidade de Tecnologia da Silésia, localizada na Polônia, foi a organização que mais publicou sobre o assunto, entretanto, outras instituições apresentam números próximos à essa Universidade.

Analisando os artigos que possuem um maior número de citações e os autores que mais publicaram sobre o tema desse estudo, foi possível perceber que apenas dois dos autores que mais publicaram no período analisado, estão entre os doze artigos mais citados. Isso mostra que, as publicações com maior número de citações, que possivelmente são as de maior referência na área pesquisada, não possuem como autores aqueles que mais publicam sobre essa ferramenta FMEA. Portanto, autores que possuem muitas publicações não são, necessariamente, aqueles que apresentam artigos de maior relevância para determinado tema de pesquisa.

Desse modo, os resultados desta pesquisa mostraram que o tema pesquisado é emergente, tendo poucos estudos publicados sobre a ferramenta na última década. Apesar disso, é necessário que novas produções científicas relacionadas ao tema sejam produzidas, pois como afirma Horita (2004), a utilização do FMEA implica em minimização das chances do produto ou processo falhar, ou seja, a partir da aplicação da ferramenta, contribui-se diretamente, na dimensão confiabilidade.

$\mathrm{RC}: 63222$

Disponível em: https://www.nucleodoconhecimento.com.br/engenharia-de-producao/efeitos-defalha 


\section{REFERÊNCIAS BIBLIOGRÁFICAS}

BIALY, Witold; RUZBARSKY, Juraj. Breakdown cause and effect analysis, case study. Managemente Sistems in Production Engineering, v. 26, ed. 2, p. 83-87, 2018.

CARBONE, T.A.; TIPPETT, D.D. Project risk management using the Project risk FMEA. Engineering Management Journal, v.16, n.4, p. 28-35, 2004.

ESPÓSITO, T. Gestão de Risco em Obras Geotécnicas em Mineração: Aplicação em Barragens de Mineração. VIII Congresso Brasileiro de Geotecnia Ambiental e VII Congresso Brasileiro de Geossintéticos, Brasília: UNICEUBE, 2015.

FERENHOF, H. A.; FORCELLINi, F. A.; VARVAKIS, G. Lições aprendidas: agregando valor ao gerenciamento de projetos. Revista de Gestão e Projetos, v. 4, n. 3, p. 197209, 2013.

GERHARDT, T.E.; SILVEIRA, D.T. Métodos de Pesquisa. UFRGS, ed. 1, 2009.

KAPLAN, S.; HAIMES, Y.Y.; LAMBERT, J.H. Fitting hierarchical holographic modeling into the theory of scenario structuring and a resulting refinement to the quantitative definition of risk. Risk Analysis, v.21, n.5, p. 807-819, 2001.

KUMAR, R. L. Managing risks in IT projects: an options perspective. Information and Management, v.40, p. 63-74, 2002.

PEETERS, J.F.W.; BASTEN R.J.I.; TINGA, T. Improving failure analysis efficiency by combining FTA and FMEA in a recursive manner. Reliability Engineering and System Safety, v.172, p. 36-44, 2018.

PEROBELLI, F. F. C. Um modelo para gerenciamento de riscos em instituições não financeiras: aplicação ao setor de distribuição de energia elétrica no Brasil. Tese de Doutorado, USP/FEA. São Paulo, 2004. 
PHILBIN, Simon P. Developing na Integrated Approach to System Safety Engineering. Engineering Management Journal, v. 22, ed. 2, p. 56-67, 2010.

PIURCOSKY, F. P. et al. Gerenciamento de riscos aplicado em um projeto de uma linha de produção. III Simpósio In- ternacional de Gestão de Projetos e II Simpósio Internacional de Inovação e Sustentabilidade, São Paulo: UNINOVE, 2014.

PUENTE, J.; PINO, R.; PRIORE, P.; FUENTE, D. A decision support system for applying failure mode and effects analysis. The International Journal of Quality \& Reliability Management, v.19, p.137-150, 2002.

RABECHINI, J.R.; Carvalho, M. M. Relacionamento entre gerenciamento de risco e sucesso de projetos. Production Journal, v. 23, n. 3, p. 570-581, 2013.

SAMAT, H. Abdul; KAMARUDDIN, S.; AZID, I. Abdul; Integration of overall equipment effectiveness (OEE) and reliability method for measuring machine effectiveness. South African Journal of Industrial Engineering, v. 23, ed. 1, p. 92-113, 2012.

SENA, E. A. et al. Gerenciamento de riscos aplicado a um projeto de revestimento de tubos para extração de óleo e gás. III Simpósio Internacional de Gestão de Projetos e II Simpósio Internacional de Inovação e Sustentabilidade, São Paulo: UNINOVE, 2014.

STADNICKA, D. Setup analysis: Combining smed with other tools. Management and Production Engineering Review, v.6, n.1, p. 36-50, 2015.

TRAMMELL, S.R.; LORENZO, D.K.; DAVIS, B.J. Integrated hazard analysis: Using the strengths of multiple methods to maximize the effectiveness. Professional Safety, v.49, n.5, p. 29- 37, 2004.

ZHANG, Yang; ANDREWS, John; REED, Sean. et al. Maintenance processes modelling and optimisation. Reliability Engineering and System Safety, v.6, ed. 1, p. 150-160, 2017. 
Enviado: Março, 2020.

Aprovado: Outubro, 2020.

RC: 63222

Disponível em: https://www.nucleodoconhecimento.com.br/engenharia-de-producao/efeitos-defalha 